\title{
Aparato educacional para estudo da queda livre com análise do movimento ${ }^{+*}$
}

\author{
Denicezar Angelo Baldo ${ }^{1}$ \\ José Luiz Antunes Almeida ${ }^{2}$ \\ José Martins Oliveira Jr. ${ }^{3}$ \\ Norberto Aranha 4 \\ Waldemar Bonventi $\mathrm{Jr}^{5}$ \\ Universidade de Sorocaba \\ Sorocaba - SP
}

\section{Resumo}

Este artigo apresenta um projeto experimental para a medição eletrônica da queda de objetos em queda livre para fins educacionais. $O$ aparelho é constituído por um tubo de PVC, dentro das quais os objetos são abandonados. Sensores de movimento foram colocados no interior do tubo, a distâncias regulares. Objetos passando por eles disparam um sinal, que é coletado, processado pela plataforma Arduino e enviado via porta serial para um módulo XBEE. Este envia dados remotamente para outro módulo XBEE conectado a uma porta USB do computador, onde um programa calcula a aceleração do movimento e serve como interface do usuário. Uma solução original foi concebida para evitar a interferência entre os sensores. Como estudo de caso, as medidas da aceleração gravitacional foram feitas com objetos de diferentes geometrias. Foram utilizados os modelos sem e com resistência do ar e obtido o coeficiente de arrasto.

Palavras-chave: Detecção óptica; Movimento de objetos; Ensino.

\footnotetext{
${ }^{+}$Educational apparatus for the freefall study with motion analysis

* Recebido: abril de 2016. Aceito: junho de 2016.

${ }^{1}$ E-mail: denicezar.baldo@edu.uniso.br

2 E-mail: jose.luiz@prof.uniso.br

3 E-mail: jose.oliveira@prof.uniso.br

${ }^{4}$ E-mail: norberto.aranha@prof.uniso.br

5 E-mail: waldemar.bonventi@prof.uniso.br
} 


\begin{abstract}
This paper presentes an experimental Project for the electronic measurement of free falling objects for educational purposes. The apparatus consists of a PVC pipe, within which the objects are released. Switching sensors were placed inside the tube at regular distances. Objects passing through them trigger a signal, which is collected, processed by the Arduino platform and sent via serial port to a XBEE module. This sends data remotely to another XBEE module attached to a USB port in a computer, where a program calculates the acceleration of movement and serves as user interface. An original solution was devised to avoid interference among sensors. As case study, measures of the gravitational acceleration were made with objects of different geometries. Models with and without air resistance were employed and drag coefficient was obtained.
\end{abstract}

Keywords: Optical sensing; Movement of objects; Teaching.

\title{
I. Introdução
}

Cursos de Engenharia apresentam em sua estrutura curricular disciplinas de Física, Cálculo, Cálculo Numérico e, em particular, nos cursos de Engenharia de Controle e Automação, Computação e Elétrica, disciplinas da área de eletricidade e programação de computadores. Normalmente, tais disciplinas agregam aulas práticas, como experimentos, projetos e desenvolvimento de programas. Um dos experimentos básicos que podem ser utilizados é o de "queda livre", em que é possível discutir questões como a obtenção da aceleração da gravidade local e a influência da resistência do ar, essenciais à formação do engenheiro. Além disso, questões relacionadas à aquisição dos dados, sua precisão, tratamento e análise são tópicos a serem considerados nos experimentos didáticos.

Ao longo do tempo, vários equipamentos têm sido apresentados para medidas da aceleração gravitacional por queda livre (CIDEPE, 2015; COLUCI, 2013; CORVELONI, 2009; FERREIRA, 2004; VASCONCELLOS, 2014). Essas montagens, em particular, apresentam algum tipo de sensoriamento, seja óptico, ímãs com ultrassom ou algum outro arranjo eletrônico.

A inclusão da plataforma de prototipagem Arduino possibilita a flexibilidade necessária para idealizar projetos relativamente simples e que envolvam controle e processamento de sinais por microcontroladores (BANZI, 2011; MCROBERTS, 2012). A plataforma Arduino oferece várias portas analógicas e digitais de conexão e um microcontrolador facilmente programável através de interface própria. 
Além disso, a possibilidade de modularizar o equipamento, separando-o em uma unidade de tomada de dados e outra de análise de dados (que se comunicam remotamente), torna-o mais flexível, acomodando-o melhor em espaços de laboratório onde alunos possam ficar próximos à atividade experimental. Assim, tecnologias de comunicação como Wifi, Bluetooth ou ZigBee estão cada vez mais acessíveis, possibilitando diferentes configurações espaciais dos equipamentos (XBEE ${ }^{\mathrm{TM}}$, 2015; ZIGBEE, 2015)

Neste trabalho optamos por utilizar o experimento de queda livre por ser um estudo clássico discutido nos livros didáticos, com o valor da aceleração gravitacional já bem estabelecido (HALLIDAY, 2013; TIPLER, 2009; YOUNG, 2007). Para este estudo apresentamos um modo de automação do experimento de queda livre, com a inclusão de sensores ópticos para a interrupção e o tratamento do sinal por técnicas eletrônicas e de programação. Com os dados, foi analisada a influência da resistência do ar durante a queda e os resultados comparados com o modelo em que tal resistência é desprezível. Foram utilizados um prumo de construção civil e esferas como objetos de queda.

\section{Queda livre}

Abordagens do problema da queda livre são encontradas nos livros de física fundamental que abordam os conceitos da mecânica clássica (HALLIDAY, 2013; TIPLER, 2009; YOUNG, 2007). Supondo-se que a única força atuando sobre um corpo durante sua queda seja o peso $\mathbf{P}=m \mathbf{g}$, sendo $\mathbf{g}$ a aceleração gravitacional da Terra, a resultante é

$$
m a=m g
$$

Escrevendo na forma de equação diferencial,

$$
m d^{2} y / d t^{2}=m g
$$

sendo $y$ a posição do corpo em relação à vertical, escolhendo-se como referência o ponto de partida do corpo $(y=0)$.

A solução da equação (2) se dá integrando-se duas vezes a equação diferencial de segunda ordem em relação a $t$. Este procedimento gera duas constantes de integração que serão determinadas pelas condições iniciais, resultando na equação do movimento com aceleração constante (3)

$$
y(t)=y_{0}+v_{0} t+1 / 2 g t^{2}
$$

sendo $y_{0}$ a posição inicial do corpo, $v_{0}$ sua velocidade inicial e $g$ a intensidade da aceleração gravitacional. No caso do corpo abandonado e sujeito apenas à atração gravitacional, $v_{0}=0$.

Coletando-se a posição e o tempo de passagem através de cada sensor, é possível realizar o ajuste da função (3) e calcular seus parâmetros. 


\section{Resistência do ar}

Em um corpo em queda livre, além do peso $P=m g$ que o move para baixo, existe a força contrária de resistência do ar. Esta força é proporcional à densidade $\rho$ do fluido dentro do qual o corpo se desloca, no caso o ar, à área $A$ da seção de choque do corpo que está cisalhando o fluido e, mais intensamente, à velocidade $v$ de deslocamento do corpo em relação ao fluido e à viscosidade dinâmica $\mu$ do fluido. Esta última fica implícita em uma grandeza denominada coeficiente de arrasto $C_{D}$ que depende ainda da geometria do corpo.

A força resultante durante a queda se torna o peso, descontado: i) desta força de resistência (força de arrasto), que contempla as grandezas acima mencionadas, e ii) da força de empuxo, pelo fato de o corpo estar imerso em fluido.

$$
F=P-F_{\mathrm{D}}-E=m g-1 / 2 C_{\mathrm{D}} \rho_{\mathrm{FLU}} A v^{2}-\rho_{\mathrm{FLU}} V g
$$

Como consequência, o movimento é acelerado, porém o aumento da velocidade diminui a força resultante (logo, esta aceleração), até que haja um equilíbrio entre $P-E$ e $F_{\mathrm{D}}$. $\mathrm{O}$ corpo passa a cair em velocidade constante devido ao término da aceleração. Esta velocidade limite é denominada velocidade terminal e pode ser determinada pela eq. (4), fazendo-se $m=$ $\rho_{\text {CORPO }} V$ e $P-F_{\mathrm{D}}-E=0$, resultando em

$$
v^{2}=2\left(\rho_{\mathrm{CORPO}}-\rho_{\mathrm{FLU}}\right) V g /\left(C_{\mathrm{D}} \rho_{\mathrm{FLU}} A\right)
$$

No entanto, para a queda de corpos sólidos e líquidos em meios gasosos, $\rho_{\mathrm{CORPO}} \gg$ $\rho_{\text {FLU, }}$ de modo que a velocidade terminal fica

$$
v_{T}=\sqrt{2 m g /\left(C_{D} \rho_{F L U} A\right)}
$$

A expressão para o movimento é obtida da eq. (4) desprezando-se o empuxo e fazendo-se $b=1 / 2 C_{\mathrm{D}} \rho_{\text {FLU }} A$ :

$$
m \frac{d v}{d t}=m g-b v^{2}
$$

A solução de (7) é dada pela separação de variáveis

$$
\frac{d v}{g d t}=\left[1-\frac{b}{m g}\right] v^{2} \rightarrow \frac{d v}{\left[1-\frac{b}{m g} v^{2}\right]}=g d t
$$

integrando-se

$$
\int \frac{d v}{1-\frac{b}{m g} v^{2}}=\int g d t
$$

com as substituições $z=\frac{b v}{m g}, d z=b \frac{d v}{m g}$ é obtida uma integral conhecida

$$
\frac{m g}{b} \int \frac{d z}{1-z^{2}}=g t+C
$$


cuja solução à esquerda, verificada em tabelas, é $\operatorname{arctanh}(z)$. Assim, a solução para a velocidade se torna

$$
v(t)=v_{T} \tanh \left(\frac{g}{v_{T}}\right)+v_{0}
$$

No caso de objeto abandonado, $v_{0}=0$. Nestas condições, integrando-se novamente e usando $\int \tanh (x) d x=\ln (\cosh (x))+C$, a distância percorrida na queda é dada por

$$
y(t)=\frac{v_{T}{ }^{2}}{g} \ln \left(\cosh \left(\frac{g t}{v_{T}}\right)\right)+y_{0}
$$

A eq. (9) é que foi utilizada no ajuste dos parâmetros da queda com resistência do ar, uma vez que $y_{0}=1,0 \mathrm{~m}$ refere-se à altura do primeiro sensor (passagem em $t=0$ ).

\section{Coeficiente de arrasto}

Em geral, o coeficiente de arrasto é determinado experimentalmente e modelos em função da geometria do corpo são produzidos (FOX, 2010). Questões como a disposição volumétrica do corpo e sua orientação enquanto cai implicam em discussões sobre como o fluido cisalhado escoa ao longo do corpo, diferenças de pressão que podem existir e a rugosidade da superfície do mesmo. O coeficiente de arrasto está relacionado com a força resistente ao movimento $\left(F_{\mathrm{D}}\right)$, a densidade do fluido no qual o corpo se desloca, a área da secção frontal do corpo e a velocidade relativa entre o corpo e o fluido (eq. 4). A força de arrasto total é a soma do arrasto de pressão, relacionado à secção frontal do corpo, e o arrasto de atrito, relacionado ao escoamento pelas laterais do corpo (FOX, 2010). Por exemplo, o arrasto puramente devido ao atrito ocorre no caso de uma placa deslocando-se paralelamente ao fluido. $\mathrm{O}$ arrasto puramente devido à pressão ocorre ao se considerar a placa deslocando-se perpendicularmente ao fluido. Porém, o coeficiente de arrasto passa a ser uma função apenas do número de Reynolds $(R e)$. Esta quantidade adimensional caracteriza efeitos de turbulência no escoamento do fluido em torno do corpo. O número de Reynolds pode ser calculado por

$$
\operatorname{Re}=\rho \phi v / \mu
$$

em que $\rho$ é a densidade do fluido que circunda o corpo de diâmetro $\phi$ que se desloca à velocidade $v$. A viscosidade $\mu$ do fluido dificulta o escoamento e a formação de turbulência. O número $R e$ é adimensional e o regime de escoamento turbulento se torna bem estabelecido com Re > 4000 (FOX, 2010; AGUIAR, 2004).

\section{Aparato experimental}

O esquema do sensoriamento utilizado consistiu na instalação de três pares emissorsensor óptico nas paredes de um tubo, percorrido por dentro por um objeto (Figura 1). A passagem do objeto pelo par emissor-sensor interrompe o fluxo luminoso entre eles, gerando em 
consequência uma interrupção do sinal. O sinal de cada par emissor-sensor é transmitido a um circuito astável 6 que os envia à plataforma Arduino. Conectada a ela, um módulo XBEE transmite remotamente os sinais ao módulo remoto XBEE-PRO que contém uma interface USB, acoplado a um computador, que fará a análise dos sinais através de um programa especialmente desenvolvido para este fim.

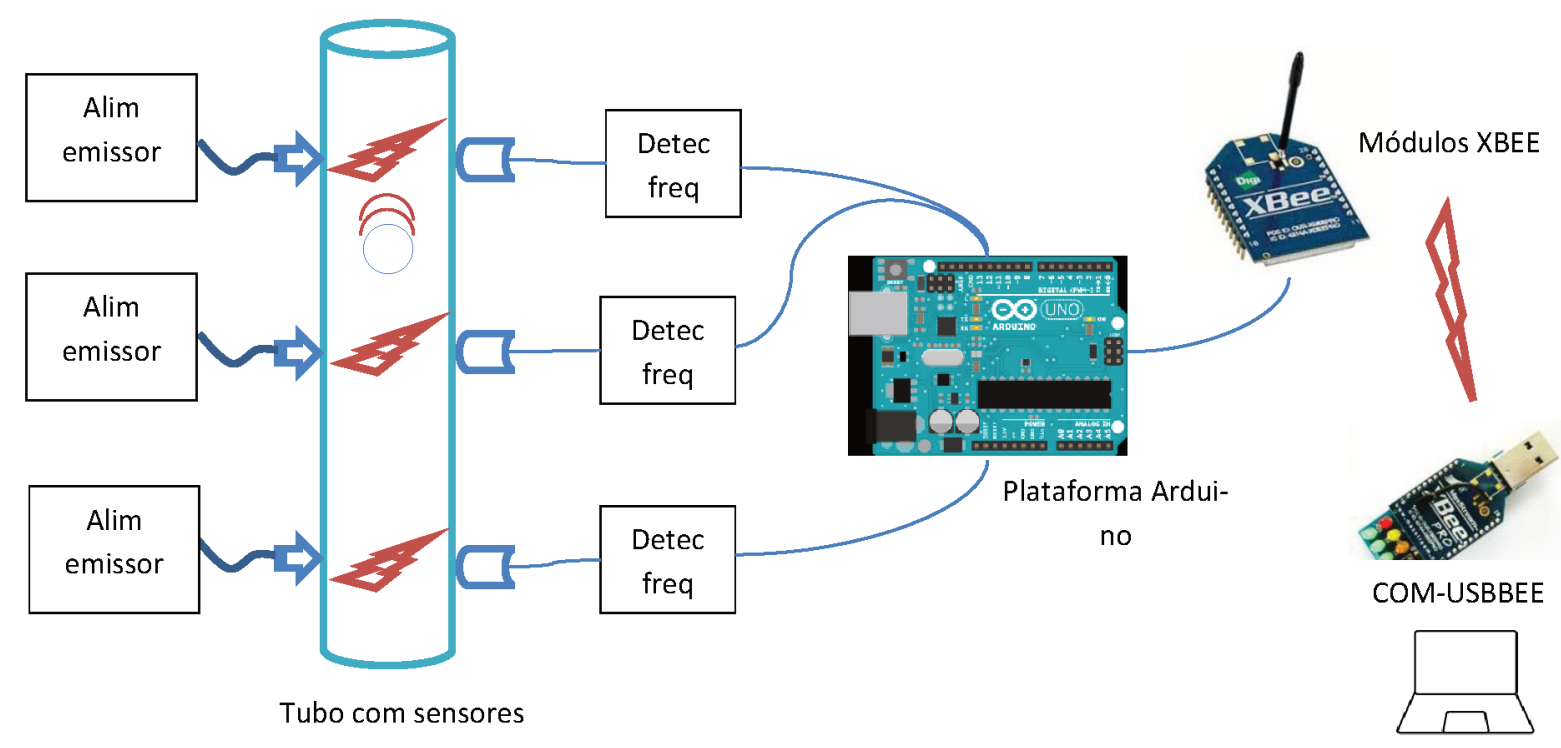

Fig. 1 - Diagrama dos componentes do sensoriamento.

Fisicamente, foi concebido um suporte de madeira no qual se adaptou um flange de PVC utilizado em conexões de água com tubos de $60 \mathrm{~mm}$ de diâmetro. A este flange, foi conectado um tubo de PVC, de mesmo diâmetro, previamente cortado com um comprimento de cerca de $1 \mathrm{~m}$. Ao longo do tubo foram instalados três conjuntos emissor-sensor, cada par em posição diametralmente oposta, sendo o primeiro conjunto logo na entrada do tubo em sua parte superior, o segundo a meio curso, distante $500 \mathrm{~mm}$ do anterior e o terceiro ao final do percurso, pouco antes do objeto lançado atingir o solo, também a $500 \mathrm{~mm}$ do anterior (Fig. 2).

O conjunto emissor-sensor foi composto de um diodo emissor infravermelho (de comprimento de onda dominante $940 \mathrm{~nm}$ e tensão de trabalho de 1,27 a 1,4 V) e um fototransistor (tensão de trabalho de $2,7 \mathrm{~V}$ a $5,5 \mathrm{~V}$, consumo de corrente de $1,5 \mu \mathrm{A}$, frequência de trabalho de $38 \mathrm{kHz}$ e ângulo de recepção de $45^{\circ}$ ). Houve o cuidado de alinhar horizontalmente o sensor e o emissor em cada trecho do tubo (Fig. 2). Além dos LEDs e foto-transistores NPN, foram conectados resistores de polarização para proteger os LEDs e adequar o acionamento dos foto-transistores. Estes elementos foram montados em uma placa universal de circuito impresso, sendo o acesso feito via conectores. Esta placa foi conectada a uma plataforma Arduino Uno R3, e sobre ela, um ProtoShield BR. Basicamente, esse shield é uma proto-

\footnotetext{
${ }^{6}$ Circuito astável é aquele que comuta dois estados de duração respectiva $T_{1}$ e $T_{2}$.
} 
board na qual foi montada um módulo XBEE para comunicação serial com o PC (no detalhe da Fig. 2), para transmitir os sinais da base do dispositivo ao computador.

No computador foi utilizado um módulo XBEE-PRO, montado em uma placa de interface COM-USB-BEE com conexão USB (Fig. 1). O módulo XBEE-PRO, conectado ao $\mathrm{PC}$, foi configurado para receber a comunicação enviada pelo módulo XBEE, transformando as informações recebidas em sinais seriais no protocolo USB e as envia a uma porta serial virtual criada no PC. Estes sinais são utilizados pelo programa hospedeiro de monitoramento e controle no computador, via conexão serial com o módulo XBEE colocado junto ao dispositivo físico de queda livre.

As Fig. 3 e 4 mostram, respectivamente, o circuito de alimentação do sensor e o circuito de tratamento do sinal recebido do foto-transistor. Conforme já citado, o Arduino utilizado foi o Uno R3 com um shield portando um módulo XBEE. O programa implementado no Arduino conta o tempo a partir do instante em que o sistema é energizado, através da função millis() que registra o instante da interrupção de cada um dos três sensores do tubo, enviando os valores pela porta serial, que os comunica ao PC remotamente através dos módulos XBEE (Fig. 1). Assim, os tempos medidos foram dados em milissegundos.

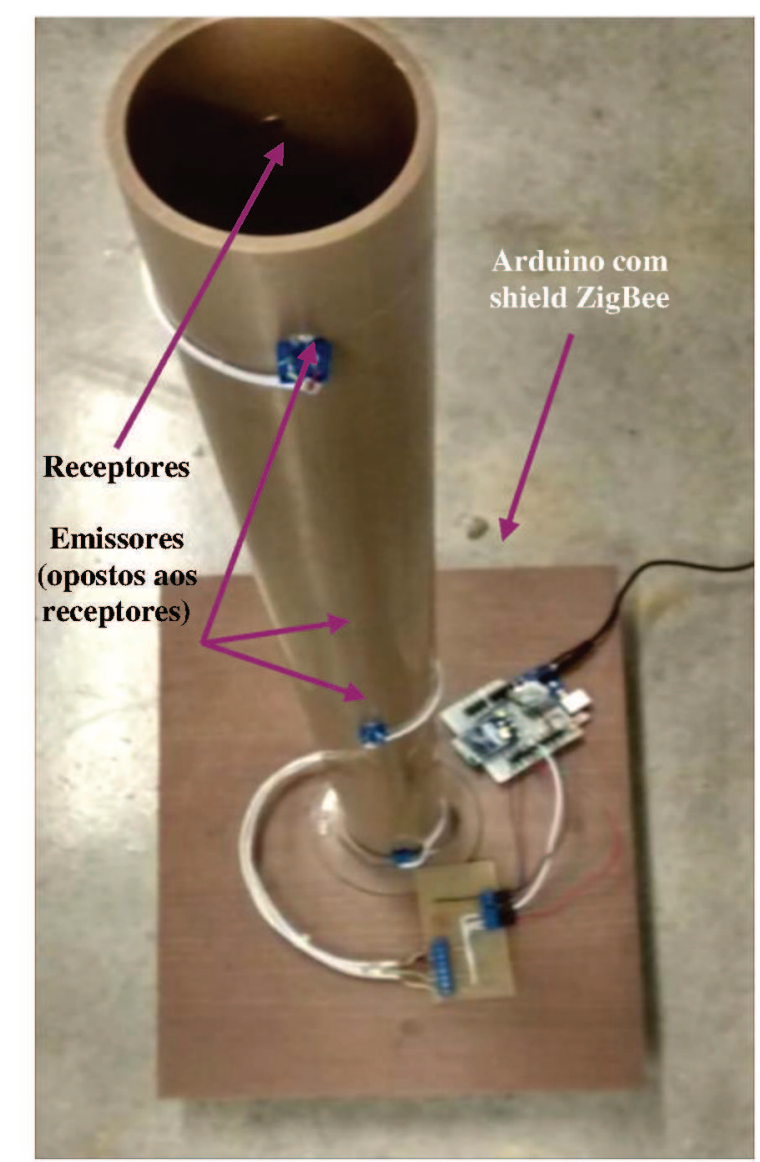

Fig. 2 - Tubo de lançamento, vista superior. 


\section{Experimentos}

Inicialmente, as placas são energizadas e o módulo COM-USBEE inserido no computador. Executa-se o programa que coleta os dados e configura-se a porta, velocidade e parâmetros de comunicação. O operador aciona o botão de abertura da porta de comunicação no programa e depois posiciona o objeto alguns centímetros acima da entrada superior do tubo, abandonando-o em queda livre. Os dados coletados dos tempos de passagem pelos sensores foram expressos no programa em milissegundos.

Foram lançados a partir da entrada superior do tubo da Figura 2 uma bolinha de pingpong, uma esfera de mouse e um prumo cilíndrico com ponta (Fig. 5). Os sinais foram captados pelos sensores e os tempos de passagem mostrados pela tela do software de comunicação. Para cada objeto, foram efetuados 10 lançamentos. Na Tabela I, observa-se o intervalo de tempo em milissegundos da passagem do objeto desde o primeiro sensor (no qual se considera $\left.t_{0}=0\right)$.

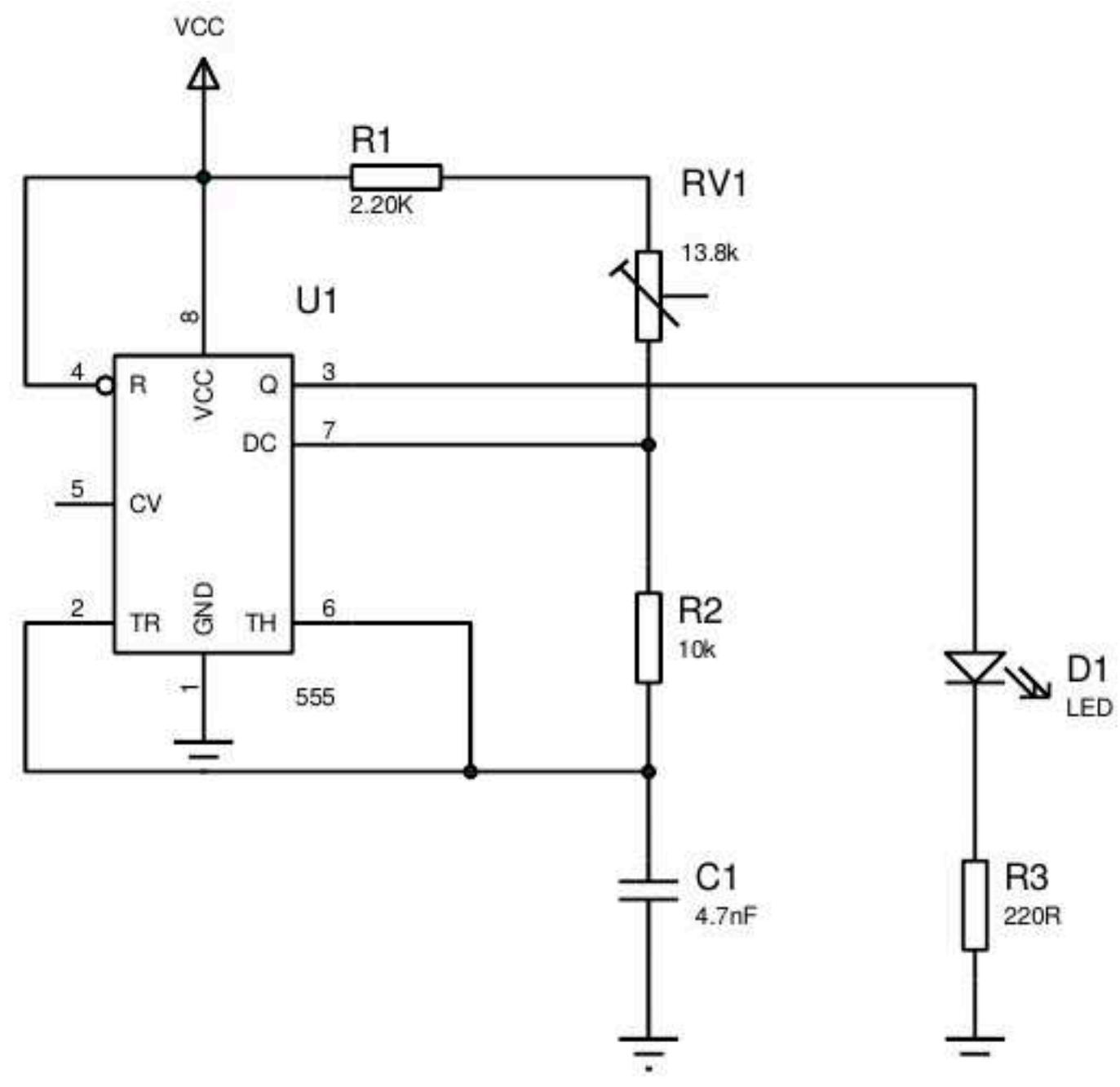

Fig. 3 - Circuito para alimentar o diodo emissor. 


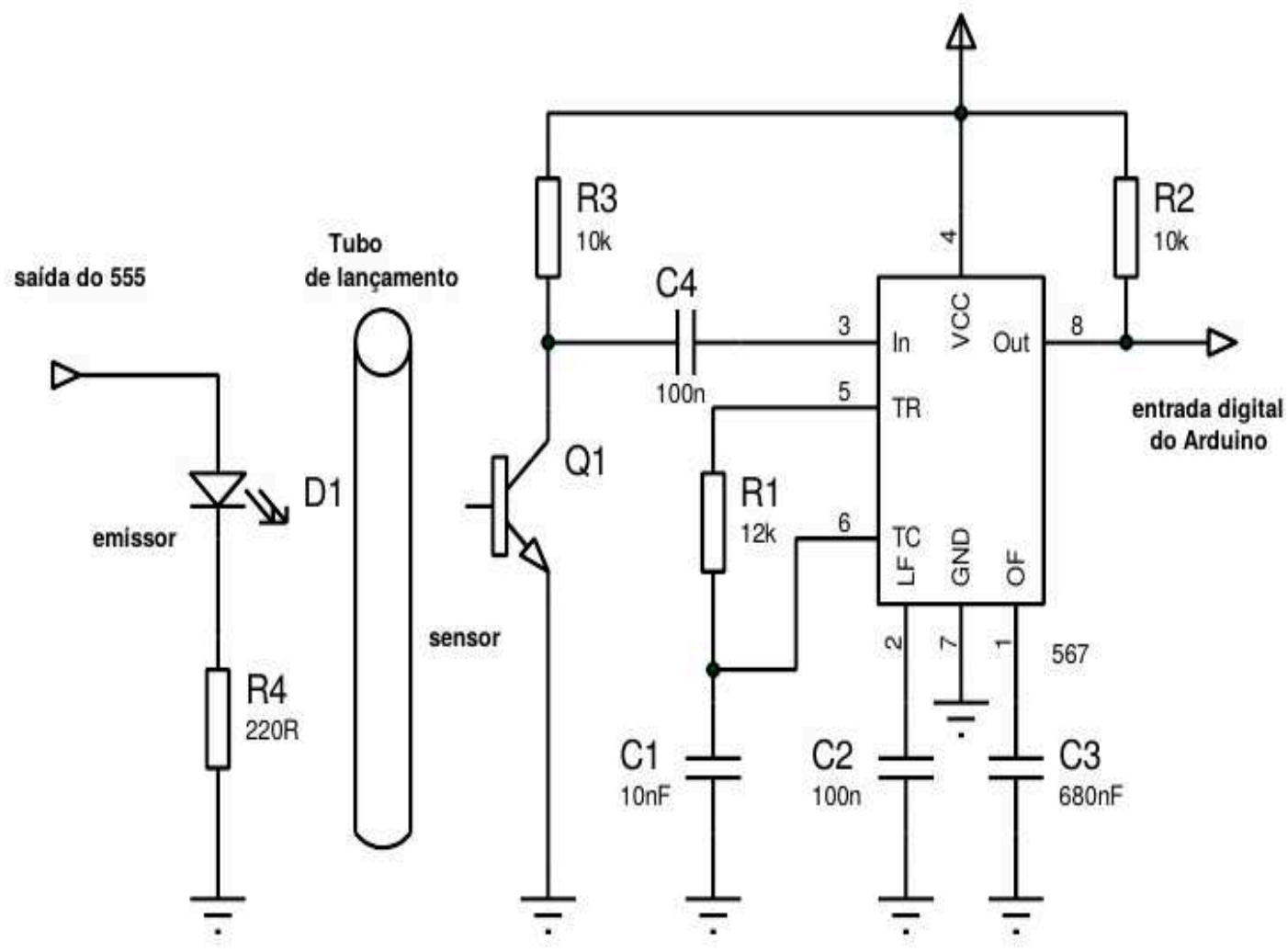

Fig. 4 - Circuito detector de frequência.

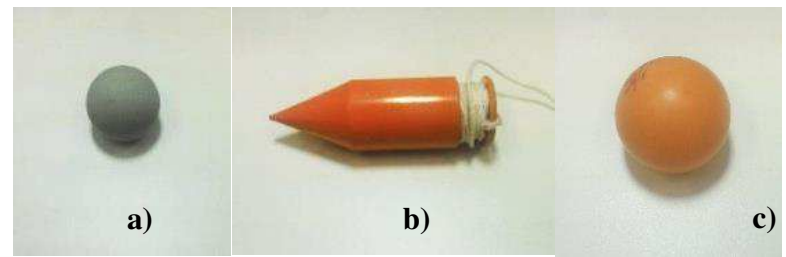

Fig. 5 - Objetos lançados: a) esfera de mouse, b) prumo cilíndrico, c) bolinha de ping-pong.

As massas e dimensões dos objetos são dadas a seguir: a) esfera de mouse: massa $m$ $=(31,48 \pm 0,01) \mathrm{g}$; diâmetro $\phi=(22,00 \pm 0,05) \mathrm{mm} ; \mathrm{b})$ prumo cilíndrico: massa $m=(123,00 \pm 0,01) \mathrm{g}$; diâmetro $\phi=(22,0 \pm 0,05) \mathrm{mm}$; comprimento $L=(62,1 \pm 0,5) \mathrm{mm}, \mathrm{c})$ bolinha de ping-pong: massa $m=(2,74 \pm 0,01) \mathrm{g}$; diâmetro $\phi=(4,00 \pm 0,05) \mathrm{mm}$

Foram tabulados os dados e obtidas as médias dos tempos de passagem de cada objeto pelos sensores. Um ou dois valores em cada conjunto ficaram fora da variação esperada (outliers) talvez devido a alguma falha de comunicação no sistema. Estes valores estão riscados na Tabela I. 
Tabela I: Tempos de passagem pelos sensores de cada objeto e aceleração gravitacional calculada (outliers aparecem riscados na tabela).

\begin{tabular}{ccc|cc|cc}
\hline \multirow{2}{*}{ lance } & \multicolumn{2}{c|}{ Bolinha de ping-pong } & \multicolumn{2}{c|}{ Bolinha de mouse } & \multicolumn{2}{c}{ Prumo cilíndrico } \\
\cline { 2 - 6 } & $\boldsymbol{\Delta} \mathbf{t}_{\mathbf{1}}(\mathbf{m s})$ & $\boldsymbol{\Delta \mathbf { t } _ { \mathbf { 2 } } ( \mathbf { m s } )}$ & $\boldsymbol{\Delta \mathbf { t } _ { \mathbf { 1 } } ( \mathbf { m s } )}$ & $\boldsymbol{\Delta \mathbf { t } _ { \mathbf { 2 } } ( \mathbf { m s } )}$ & $\boldsymbol{\Delta} \mathbf{t}_{\mathbf{1}}(\mathbf{m s})$ & $\boldsymbol{\Delta t}_{\mathbf{2}}(\mathbf{m s})$ \\
1 & 264 & 421 & 241 & 370 & 255 & 384 \\
2 & 315 & 483 & 238 & 366 & 261 & 391 \\
3 & 271 & 428 & 244 & 373 & 251 & 381 \\
4 & 270 & 429 & 242 & 371 & 252 & 382 \\
5 & 268 & 424 & 242 & 371 & 490 & 618 \\
6 & 268 & 423 & 242 & 370 & 279 & 408 \\
7 & 274 & 433 & 241 & 570 & 262 & 392 \\
8 & 270 & 426 & 242 & 371 & 259 & 388 \\
9 & 273 & 431 & 239 & 367 & 254 & 384 \\
10 & 269 & 423 & 242 & 371 & 258 & 387 \\
\hline Média & 269,7 & 426,4 & 241,3 & 370,0 & 256,5 & 386,1 \\
Desvio padrão & 3,0 & 4,1 & 1,7 & 2,1 & 4,1 & 3,9 \\
\hline
\end{tabular}

\section{Ajuste do modelo sem resistência do ar}

O primeiro ajuste realizado foi sem considerar a resistência do ar, pela eq. (3) e a partir das médias da Tabela I, obtendo-se os resultados mostrados na Tabela II. Derivando-se a eq (3), obtém-se a velocidade e, igualando-se a zero, obtém-se o instante em que a esfera foi lançada. Os resultados mostrados na Tabela II estão coerentes com as dimensões do aparato experimental.

Obviamente, dentro da precisão do arranjo físico do experimento, a aceleração da gravidade não seria expressa com tantas casas decimais, mas ficaria muito próxima do seu valor teórico, demonstrando a validade do dispositivo de queda livre.

Tabela II: Parâmetros obtidos por interpolação do modelo sem resistência do ar.

\begin{tabular}{ccccccc}
\hline Objeto & $y_{0}(\mathrm{~m})$ & $v_{0}(\mathrm{~m} / \mathrm{s})$ & $g\left(\mathrm{~m} / \mathrm{s}^{2}\right)$ & $t_{\mathrm{INI}}(\mathrm{s})$ & $y_{\mathrm{INI}}(\mathrm{m})$ & $v_{\mathrm{FIN}}(\mathrm{m} / \mathrm{s})$ \\
B. ping-pong & 1 & $-1,001$ & $-6,261$ & $-0,161$ & 1,081 & $-3,680$ \\
B. mouse & 1 & $-0,890$ & $-9,800$ & $-0,091$ & 1,040 & $-4,516$ \\
$\quad$ Prumo & 1 & $-0,645$ & $-9,035$ & $-0,071$ & 1,023 & $-4,087$ \\
\hline
\end{tabular}

\section{Ajuste do modelo com resistência do ar proporcional ao quadrado da velocidade}

Conforme discutido anteriormente, o modelo que se aplica ao regime de queda livre com resistência do ar é o dado pela eq. (9), onde os parâmetros de ajuste são a posição $y_{\mathrm{INI}}$ de onde o objeto foi abandonado (acima do primeiro sensor), a aceleração gravitacional $g$, a velocidade terminal $v_{\mathrm{T}}$ e o instante $t_{\mathrm{INI}}$ de abandono do objeto. 
A exemplo da Tabela II, o ajuste foi realizado em relação aos intervalos de tempo de passagem entre os sensores $\Delta \mathrm{t}_{1} \mathrm{e} \Delta \mathrm{t}_{2}$. Como a equação (9) é não-linear nos parâmetros, o programa de ajuste teve como requisitos minimizar o desvio entre os valores de $y(t)$ da função ajustada e os $y_{\mathrm{i}}$ fixados pelas posições dos sensores, nas alturas $1,0 \mathrm{~m}, 0,5 \mathrm{~m}$ e 0,0 $\mathrm{m}$. Programas de minimização buscam o mínimo global ou ótimo, porém podem existir mínimos locais que "armadilham" a convergência iterativa. Além disso, neste caso existem apenas três dados para cada lançamento dos objetos para ajuste de quatro parâmetros. O programa utilizado foi o software livre SciDAVis (STANDISH, 2016), desenvolvido para fins de análise, visualização e ajuste de funções, a exemplo de similares comerciais. $\mathrm{O}$ algoritmo usado para ajuste foi o de Levenberg-Marquardt. A solução para obter a convergência a valores fisicamente razoáveis foi limitar a variação dos parâmetros conforme a Tabela III.

Os resultados obtidos após o ajuste do modelo com resistência do ar estão na Tabela IV $\left(t_{\mathrm{INI}}, y_{0}, v_{\mathrm{T}}, g\right)$. A velocidade final $v_{\mathrm{FIN}}$ foi obtida com o tempo de passagem no último sensor, como na Tabela II. Observa-se que as limitações impostas para convergência do algoritmo resultaram em valores fisicamente plausíveis.

Tabela III: Valores iniciais dos parâmetros para o ajuste não-linear da eq. (9).

\begin{tabular}{cccl}
\hline Parâmetro & Mínimo. & Máximo & \multicolumn{1}{c}{ Justificativa } \\
$t_{\mathrm{INI}}(\mathrm{ms})$ & -1000 & 0 & Valores orientados pelas \\
$y_{\mathrm{INI}}(\mathrm{m})$ & 1,0 & 2,0 & condições físicas dos \\
$v_{\mathrm{T}}(\mathrm{m} / \mathrm{s})$ & 0 & 300 & lançamentos. \\
$g\left(\mathrm{~m} / \mathrm{s}^{2}\right)$ & $-9,0$ & 11,0 & \\
\hline
\end{tabular}

Tabela IV: Valores finais dos parâmetros no ajuste não-linear da eq. (9).

\begin{tabular}{cccccc}
\hline Objeto & $t_{\mathrm{INI}}(\mathrm{s})$ & $\begin{array}{c}y_{0} \\
(\mathrm{~m})\end{array}$ & $\begin{array}{c}v_{\mathrm{T}} \\
(\mathrm{m} / \mathrm{s})\end{array}$ & $\begin{array}{c}g \\
\left(\mathrm{~m} / \mathrm{s}^{2}\right)\end{array}$ & $\begin{array}{c}v_{\mathrm{FIN}} \\
(\mathrm{m} / \mathrm{s})\end{array}$ \\
$\begin{array}{cccc}\text { B. ping-pong } \\
\text { B. mouse }\end{array}$ & $-0,073$ & 1,025 & 4,327 & 9,791 & 3,497 \\
Prumo & $-0,090$ & 1,040 & 49,47 & 9,840 & 4,513 \\
\hline
\end{tabular}

Na Fig. 6 estão os gráficos contendo os dados experimentais e as curvas ajustadas de acordo com os modelos sem e com resistência do ar. A altura medida refere-se à posição do sensor inferior. Valores negativos da altura são calculados como se os objetos continuassem caindo. Nota-se o efeito da resistência do ar retardando o movimento, observando-se as curvas superiores em cada gráfico. As respectivas curvas inferiores são as parábolas do modelo sem considerar a força de arrasto.

Nota-se o forte arrasto na bolinha de ping-pong devido ao seu pequeno peso $(\sim 0,03 \mathrm{~N})$ que resulta em uma aceleração efetiva de queda de $\sim 6,3 \mathrm{~m} / \mathrm{s}^{2}$, que pode ser significativamente observada antes de $1 \mathrm{~s}$ de queda. Para a bolinha do mouse, a diferença começa a 
ser significativa a partir de $2 \mathrm{~s}$ (quando já teria caído quase $20 \mathrm{~m}$ ) e, no caso do prumo, após 5 $\mathrm{s}$ temos uma diferença de $1 \mathrm{~m}$ em $120 \mathrm{~m}$, devido ao seu maior peso $(\sim 1 \mathrm{~N})$ e menor coeficiente de arrasto. Para verificar isto, adiante é calculado o coeficiente de arrasto em função da velocidade final de queda, a partir dos dados experimentais.
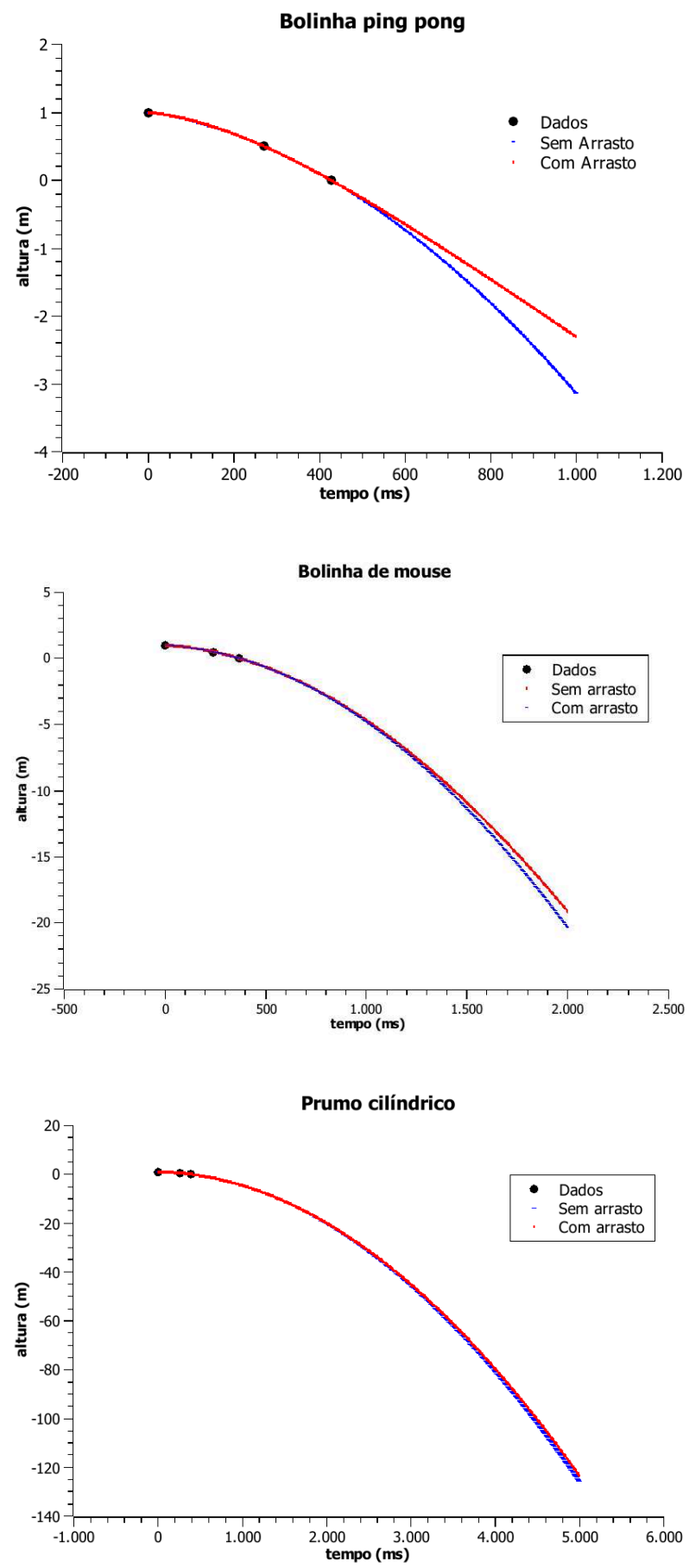

Fig. 6 - Gráficos da posição do objeto em relação aos sensores e projeção da mesma segundo os modelos sem e com resistência do ar (arrasto). 
Comparando-se as velocidades de passagem pelo segundo sensor (quase no solo ver Fig. 1 e Tabela II), nos modelos com e sem resistência do ar, percebe-se que ficaram muito próximas relativamente a cada objeto. Isto nos leva à hipótese de que o coeficiente de arrasto é suficientemente baixo, de modo a tornar os dois modelos quase indistinguíveis pela altura de queda ocorrida $(\sim 1 \mathrm{~m})$.

Na Tabela V são mostrados os resultados do número de Reynolds e do coeficiente de arrasto dos objetos lançados. A tabela está dividida em duas partes, mostrando $R e$ e $C_{\mathrm{D}}$ no final da queda (relacionados à $v_{\text {FIN }}$ ) e a previsão dos mesmos no limite $v_{\text {T. }}$ Re foi calculado pela eq. (10). Para os objetos esféricos, $C_{\mathrm{D}}$ pode ser diretamente obtido de gráficos ou dados experimentais (FOX, 2010; AGUIAR, 2004). Para o prumo, o coeficiente de arrasto para baixas velocidades foi estimado a partir de dados já existentes de foguetes (HOERNER, 1965) em virtude da semelhança geométrica, mas no limite $v_{\mathrm{T}}$ foi realizado o cálculo pela eq. (6).

TABELA V: Velocidade, $\mathrm{n}^{\circ}$ de Reynolds e coeficiente de arrasto ao final da queda e no limite $v_{\mathrm{T}}$.

\begin{tabular}{l|lll|lll}
\hline Objeto & $v_{\text {FIN }}(\mathrm{m} / \mathrm{s})$ & $R e$ & $C_{D}$ & $v_{\mathrm{T}}(\mathrm{m} / \mathrm{s})$ & $R e$ & $C_{D}$ \\
\hline B. ping-pong & 3,497 & 9947 & $\sim 0,4$ & 4,327 & 12065 & $\sim 0,4$ \\
B. mouse & 4,514 & 7133 & $\sim 0,4$ & 49,47 & 78186 & $\sim 0,5$ \\
Prumo & 4,490 & 7095 & $0,2-0,4^{*}$ & $-158,3$ & 250130 & $\sim 0,2^{\times}$ \\
\hline \multicolumn{7}{r}{ * WHITE }
\end{tabular}

* WHITE (2011) e HOERNER (1965) × eq. (6)

\section{Discussão e conclusões}

O objetivo deste trabalho foi atingido, no sentido de produzir um equipamento experimental para medidas de grandezas físicas da queda livre e o método de aquisição de dados, através de sensores ópticos.

Para uma esfera lisa, o coeficiente de arrasto $C_{\mathrm{D}}$ é inversamente proporcional a $R e$ de acordo com a teoria de Stokes (FOX, 2010; WHITE, 2011) até $R e \sim 1$ e decresce menos fortemente $(\sim 1 / \sqrt{ } R e)$ até $R e \sim 1000$. A partir daí, $C_{\mathrm{D}}$ se torna aproximadamente linear, variando pouco, próximo a 0,42 , até cerca de $R e \sim 2.10^{5}$, quando ocorre a crise do arrasto (AGUIAR, 2004), ficando próximo a 0,1 . Tal comportamento deve-se à mudança dos efeitos de pressão na frente e atrás da esfera e ao modo de escoamento da camada limite. Gráficos do coeficiente de arrasto de uma esfera lisa em função do número Re podem ser vistos p. ex. em AGUIAR (2004), FOX (2010) e WHITE (2011).

No caso de objetos com geometrias mais complexas, $C_{\mathrm{D}}$ é determinado tanto analiticamente quanto experimentalmente em túneis de vento e simulações. Por exemplo, para o prumo utilizado (Figura 5 b), não há um valor de $C_{\mathrm{D}}$ especificamente tabelado, pois o mesmo é composto de um cone na extremidade de um cilindro. Um cone apresenta $C_{\mathrm{D}} \sim 0,5$ (com 
variações em relação ao ângulo de abertura) enquanto que um cilindro longitudinalmente apresenta $C_{\mathrm{D}} \sim 0,8$. Hoerner (1965) apresenta resultados de $C_{\mathrm{D}}$ para cilindros com cone, para protótipos de foguetes, que permitiu estimar este valor para o prumo em baixa velocidade, no caso ao atingir o solo logo após o sensor inferior. Foi possível calcular o coeficiente de arrasto do prumo no caso limite através da eq. (6) com resultado satisfatório.

Os dados experimentais mostram uma divergência muito pequena na altura de queda devida aos dois modelos empregados (com e sem resistência do ar), que só se evidenciaram com a extrapolação dos ajustes para tempos maiores. Uma forma de melhor visualizar o efeito da resistência do ar, particularmente para a bolinha de ping-pong, seria utilizando um tubo mais longo, pelo menos de $3 \mathrm{~m}$ de comprimento.

Quanto ao software de gerenciamento do dispositivo de queda livre, poderíamos optar por uma solução mais completa como, por exemplo, os softwares Matlab (2015) ou LabVIEW (2015) utilizado por Ferreira (2004). Porém, estas soluções são mais dispendiosas, visto que estes não são softwares livres. O desenvolvimento do software para aquisição e tratamento dos dados deste trabalho possibilitou: a) o controle da porta de comunicação, recebendo os dados apenas no instante do lançamento do objeto e b) o cálculo da aceleração gravitacional por meio de programação própria.

Além de experimentos de movimento de objetos pelo tubo explorado em física clássica, alunos de Engenharia Elétrica e de Controle e Automação podem se beneficiar do conhecimento obtido na construção de um sistema de sensoriamento como este. Neste contexto, insere-se o estudo dos sensores, do tratamento eletrônico dos sinais, dos procedimentos de comunicação e da programação envolvida, tanto do Arduino quanto da interface para o computador. O emprego do módulo de comunicação ZigBEE, além da eliminação de cabeamento (que no caso USB é restrito a poucos metros), permite a utilização de apenas um tubo, com o sistema de sensoriamento transmitindo dados a vários computadores com módulos COMUSBEE, que podem estar ao redor do aparato em um laboratório didático.

\section{Agradecimentos}

Os autores agradecem ao CNPq, projeto "Forma Engenharia" 455685/2012-6, à Fundação de Amparo à Pesquisa do Estado de São Paulo (FAPESP) - Processo 2014/21122-0 pelo apoio financeiro para a realização do projeto e à Universidade de Sorocaba.

\section{Referências}

AGUIAR, C.E.; RUBINI, G. A aerodinâmica da bola de futebol. Revista Brasileira de Ensino Física, São Paulo, v. 26, n. 4, p. 297-306, Dec. 2004.

BANZI, M. Primeiros passos com o Arduino. São Paulo: Novatec, 2011. 
CIDEPE - Produtos - Física - EQ811K Conjunto para queda de corpos, sensor, pino LM e software.

2015.

Disponível

em:

$<$ http://www.cidepe.com.br/pt/produtos/fisica/todos/conjunto-para-queda-de-corpos-sensorpino-lm-e-software-eq811k>. Acesso em: 18 mar. 2015.

COLUCI, V. R. et al. Ilustração de incertezas em medidas utilizando experimentos de queda livre. Revista Brasileira de Ensino de Física, v. 35, n. 2, p. 1-6, jun. 2013.

CORVELONI, E. P. M. et al. Utilização de máquina fotográfica digital (multi-burst) para aulas experimentais de cinemática - queda livre. Revista Brasileira de Ensino de Física, v. 31, n. 3, p. 3504.1-3504.4, set. 2009.

FERREIRA, E. S. et al. Diferença no tempo de queda entre dois objetos de massa diferente em queda livre. IC1-27.pdf. 2015. Disponível em:

<http://www.inicepg.univap.br/cd/INIC_2004/trabalhos/inic/pdf/IC1-27.pdf>. Acesso em: 18 mar. 2015.

FOX, R. W.; PRITCHARD, P. J.; MCDONALD, A. T. Introdução à mecânica dos fluidos. Rio de Janeiro: LTC, 2010. xiv, 710 p. ISBN 9788521617570 (broch.)

HALLIDAY, D.; RESNICK, R.; WALKER, J. Fundamentos de Física. 12. ed. Rio de Janeiro: Livros Técnicos e Científicos, 2013. v. 1.

HOERNER, S. F. Fluid-dynamic drag: Practical Information on Aerodynamic Drag and Hydrodynamic Resistence. 2 ed. N.J.: Midland Park, 1965.

LABVIEW. Ambiente gráfico de desenvolvimento de sistemas LabVIEW - National Instruments. 2015. Disponível em: <http://www.ni.com/labview/pt/>. Acesso em: 29 out. 2015.

LUZ, C. E. S. Criação De Sistemas Supervisórios Em Microsoft Visual C\# 2010 Express. São Paulo: Érica, 2012.

MATLAB. The Language of Technical Computing. 2015. Disponível em: <http://www.mathworks.com/products/matlab/>. Acesso em: 29 out. 2015.

MCROBERTS, M. Arduino Básico. São Paulo: Novatec, 2012.

STANDISH, R.; SciDAVis. Free Application for Scientific Data Analysis and Visualization. 2016. Disponível em: <http://scidavis.sourceforge.net/about.html>. Acesso em: mar 2016. 